\title{
REHOVOT RADIOCARBON MEASUREMENTS III
}

\section{ISRAEL CARMI}

Department of Isotope Research, The Weizmann Institute of Science, 76100 Rehovot, Israel

This list contains results obtained between 1981 and 1985. Since the first description of the laboratory (Carmi, Noter \& Schlesinger, 1971) the following changes were made. Two proportional counters are now used: 1) $0.5 \mathrm{~L}$ volume, $0.865 \pm 0.023 \mathrm{cpm}$ background, $12.830 \pm .134 \mathrm{cpm}$ NBS oxalic acid standard (old); 2) $0.25 \mathrm{~L}$ volume, $0.484 \pm 0.023 \mathrm{cpm}$ background, $6.185 \pm .123 \mathrm{cpm}$ NBS oxalic acid standard (old). The passive shield has been increased by $2 \mathrm{~cm}$ of mercury next to the counters. For anticoincidence, a modular, hand-made gas counter is used. The laboratory was transferred to the ground floor of a 7 -storey building. Data acquisition and processing are done with a scaler/buffer built at the Institute and an IBM PC computer. Samples are filled into the counters and counted for ca $1000 \mathrm{~min}$ at least twice. The sample preparation method and counter filling pressure have not been changed.

\section{ACKNOWLEDGMENT}

Thanks are due $\mathrm{S}$ Kazes for technical help in the lab.

\section{ARCHAEOLOGIC SAMPLES}

Marine Samples

Israel

\section{Kfar Samir series}

Prehistoric submerged settlement $2 \mathrm{~km} \mathrm{~S}$ of Haifa, $50 \mathrm{~m}$ offshore (Natl Grid ref 1461-2441).

\section{RT-598B.}

$4800 \pm 70$

Oak tree excavated $1 \mathrm{~m}$ below sea level (bsl). Coll 1981 by M Evron, Lab Prehist, Univ Haifa.

\section{RT-682A.}

$6470 \pm 130$

Wood from construction \#5, 4.5m bsl. Coll 1984 by A Raban, Center for Marine Studies, Univ Haifa.

\section{RT-682B.}

$$
\begin{array}{r}
6670 \pm \mathbf{1 4 0} \\
\delta^{13} C=-26.4 \% o
\end{array}
$$

Wood from construction \#3. $4.5 \mathrm{~m}$ bsl. Coll 1984 by A Raban.

\section{CAHEP series}

Samples coll during Cesarea Ancient Harbor Excavation Proj by A Raban and N Karmon. 
RT-609. Harbor

$1470 \pm 50$

Coll 1981 (Natl Grid Ref 1397-2124). Comment (AR): wood from cradle, used to lower construction stones during attempt by Emperor Anastasius to rebuild harbor.

RT-631A. Harbor/ship

$$
\begin{array}{r}
2210 \pm 190 \\
\delta^{13} C=-26.4 \% 0
\end{array}
$$

Coll 1983 from $9 \mathrm{~m}$ bsl (Natl Grid Ref 1398-2124). Comment (AR): beam from entrance to harbor in Herodian period. Sample is from quay or ship.

RT-631B. Harbor

$$
\begin{array}{r}
1970 \pm 70 \\
\delta^{13} C=-25.8 \% 0
\end{array}
$$

Coll 1983 from $6 \mathrm{~m}$ bsl (Natl Grid Ref 1398-2124). Comment (AR): wood from frames used in construction of harbor.

RT-645. Side plate

RT-653B. Rib I

RT-653C. Rib II

RT-680A. Tenon I

RT-680B. Tenon II

Coll 1983 from 2.5m bsl (Natl Grid Ref 1403-2127). Comment (AR): parts recovered from sunken ship from 1 st century AD.

RT-652. Acre

$2310 \pm 50$

Wood from sideplate of ship brought up by dredger during deepening of harbor (Natl Grid Ref 1569-2583). Coll 1983 and subm by N Karmon.

\section{RT-684. Dor ship I}

$$
\begin{array}{r}
100 \pm 100 \\
\delta^{13} C=-25.1 \% 0
\end{array}
$$

Wood from unid. sunken ship at Dor, $27 \mathrm{~km} \mathrm{~S}$ of Haifa (Natl Grid Ref 1424-2237). Coll 1983 by S Wachsmann, Dept Antiquities, Ministry Educ, from 2 to $3 \mathrm{~m}$ bsl. Comment (SW): possibly from ship that sank in 1664 .

\section{RT-686A. Dor ship II}

$$
\begin{array}{r}
1590 \pm 110 \\
\delta^{13} C=-28.2 \% 0
\end{array}
$$

Wood from Byzantine ship at Dor (Natl Grid Ref 1422-2238). Coll 1983 by S Wachsmann, from 2 to 3m bsl (Wachsmann \& Raveh, 1984). Comment (SW): ceramics suggest that ship is from 6th-7th century AD. 


\section{RT-686B. Atlit ship}

$\mathbf{9 9 0} \pm \mathbf{1 0 0}$

Wood from sunken ship in Atlit (Natl Grid Ref 1449-2346). Coll 1982 by S Wachsmann, from 2 to $3 \mathrm{~m}$ bsl.

\section{RT-710. Hahotrim ship}

$1800 \pm 100$

Wood from sunken ship in Hahotrin (Natl Crid Ref 1456-2400). $\mathrm{C}$ 1984 by $\mathrm{S}$ Wachsmann from 2 to $3 \mathrm{~m}$ bsl.

RT-681. Ram

$2100 \pm 110$

$\delta^{13} C=-27.6 \%$

Wood from bronze ram. Coll 1980 offshore of Atlit (Natl Grid Ref 1445-2348). Subm by N Karmon.

RT-707. Nahal Oren

$$
\begin{array}{r}
\mathbf{8 1 4 0} \pm \mathbf{1 3 0} \\
\delta^{13} C=-26.4 \% \text { o }
\end{array}
$$

Charcoal from prehistoric site presently $300 \mathrm{~m}$ offshore of Nahal Oren. Coll 1984 from $1 \mathrm{~m}$ bsl by E Galilee, Center for Marine Studies, Univ Haifa.

$$
\text { Italy }
$$

RT-705. Oristano

$$
100 \pm 100
$$

Wood from sunken ship, $20 \mathrm{~km} \mathrm{~N}$ of Oristano, Sardinia, $2 \mathrm{~m}$ bsl. Coll 1984 by E Galilee.

\section{Terrestrial Samples}

Israel

\section{RT-611. Olive seeds}

$320 \pm 70$

Charred olive (Olea europea) from old agric terrace in Jerusalem. Coll 1982 by G Edelstein, Dept Antiquities, Ministry Educ. Comment (GE): Canaanite pottery was found in terrace but sample is probably of secondary origin.

RT-614. Kaukab

$700 \pm 70$

Olive tree (Olea europea) from Kaukab in lower Galilee (Natl Grid Ref 1735-2496). Coll 1982 by Y Sela, Jewish Natl Fund. Comment (YS): part of tree exposed by erosion.

\section{Uvda Valley series}

Archaeol excavation in Arava valley $40 \mathrm{~km} \mathrm{~N}$ of Eilat.

a) Loc 906 (Natl Grid Ref 1468-9297). Charcoal from stone bowl 0.5m below ground surface. Coll 1980 by O Yogev, Dept Antiquities, Ministry Educ (Yogev, 1984). 
Comment (OY): open sanctuary. Sites with similar stereographic plans have not been previously known before 3rd-4th millennium BC.

b) Loc 916 (Natl Grid Ref 1465-9287). Samples from residential site. Coll 1980 by O Yogev.

RT-640A.

$4800 \pm 70$

$\delta^{13} C=-24.6 \%$

Charcoal from stone cache $1.5 \mathrm{~m}$ below ground surface.

RT-640B.

Charcoal from under secondary wall.

RT-640C.

Charcoal from implement $0.7 \mathrm{~m}$ below ground surface. Comment (OY): residential sites are known in region from 3 rd millennium $\mathrm{BC}$.

RT-648A. Shrine

$5670 \pm 90$

Charcoal from massebot shrine (Natl Grid Ref 1495-9255). Coll 1982 by U Avner, Dept Antiquities, Ministry Educ. Comment (UA): evidence for early desert habitation and cult sites (Henry, 1982; Rosen, 1984).

RT-648B. Threshing floor

$4250 \pm 50$

Charcoal from threshing floor (Natl Grid Ref 1495-9255). Coll 1982 by U Avner. Comment (UA): date suggests MBI period but find points to EBII, $400 \mathrm{yr}$ earlier. This date, together with those of samples $714 \mathrm{~A}$ and $714 \mathrm{~B}$ point to longer duration of EBII culture in desert compared to more humid regions.

\section{RT-714A. Site 9}

$$
4070 \pm 100
$$$$
\delta^{13} \mathrm{C}=-13.7 \%
$$

Charcoal from residential site (Natl Grid Ref 9683-1462) 0.6m below ground surface. Coll 1980 by U Avner and O Ilan (Dept of Antiquities, Ministry Educ).

\section{RT-714B. Site 166}

$$
\begin{array}{r}
\mathbf{3 8 5 0} \pm \mathbf{1 0 0} \\
\delta^{13} C=-18.2 \% 0
\end{array}
$$

Charcoal from residential site (Natl Grid Ref 9277-1459) $0.7 \mathrm{~m}$ below ground surface. Coll 1980 by U Avner.

\section{Dor series}

Ancient harbor $24 \mathrm{~km} \mathrm{~S}$ of Haifa (Natl Grid Ref 1425-2247). 
RT-630. Floor 1

Coll 1981 by A Raban.

\section{RT-685. Locus 101}

$3640 \pm 200$

Coll 1984 by A Raban.

\section{Zalaka series}

Tumuli tombs field in Wadi Zalaka, E Sinai (Natl Grid Ref 0884-8239) (Avner, 1984). All samples are charcoal, coll 1983 by U Avner. Comment (UA):date supports idea that appearance of tumuli should be moved back to 4 th or 5 th millennium BC.

RT-648E.

$5440 \pm 80$

$\delta^{13} C=-23.9 \%$

\section{Har Shani series}

Charcoal from open shrine $18 \mathrm{~km}$ NW of Eilat (Natl Grid Ref 13609000). Coll 1981 by U Avner. Comment (UA):evidence suggests that shrine had been in intermittent use between 4th millennium BC and Byzantine times. Date suggests that shrine had been in use by the Nabatean as late as 6 th century AD.

RT-648F.

RT-648G.

$$
\begin{array}{r}
1470 \pm \mathbf{6 0} \\
\delta^{13} C=-22.6^{0} \% 0 \\
1500 \pm \mathbf{1 7 0}
\end{array}
$$

\section{Shiqmim series}

Charcoal from Chalcolithic village near Beer Sheva (Natl Grid Ref 1170-0689). Coll 1982 by T E Levy, Negev Mus, Beer Sheva (Levy, 1983).

RT-649B.

RT-649D. Locus 412

\section{RT-650. Nahal Hemar}

Charcoal from cave in Judean Desert (Natl Grid Ref 1675-0645), from Neolithic pre-ceramic B layer which contains intact artifacts. Coll by O BarYosef, Inst Archeol, Hebrew Univ. Comment (IC): measurements in other 
labs gave following results: $6230 \pm 80 \mathrm{BC}($ PTA-3650) and $6300 \pm 70 \mathrm{BC}$ (BM-2298).

\section{RT-656. Kasr El Yahud}

$$
\begin{array}{r}
1380 \pm 180 \\
\delta^{13} C=-20.2 \% o
\end{array}
$$

Wood from common burial ground at Kasr El Yahud in Lower Jordan R, (Natl Grid Ref 2012-1386). Coll 1983 by J Zias, Dept Antiquities, Ministry Educ.

\section{Yiftahel series}

Burned bricks from excavation at Yiftahel in lower Galilee (Natl Grid Ref 1710-2405). Coll 1984 by E Brown, Dept Antiquities, Ministry Educ.

RT-702A.

$$
5570 \pm 220
$$

Sample from EBI layer.

RT-702B.

$7460 \pm 210$

Sample from Neolithic pre-ceramic layer.

\section{RT-718. Silo site}

$5540 \pm 110$

Triticum diococcum from silo in Chatcolithic site, Golan Heights (Natl Grid Grid Ref 2234-2564). Coll 1981 by C Epstein, Dept Antiquities, Ministry Educ.

\section{Other Countries}

RT-612A. Honduras del Oeste

$3540 \pm 70$

Shells (Caracolus excellens) from Santo Domingo. Coll from ancient refuse dump in 1981. Subm by M Vellos Magiolo.

RT-612B. Cacoq

$\mathbf{3 0 9 0} \pm \mathbf{5 0}$

Shells (Arca occidentalis) from Ihle a Vache, Haiti. Coll 1982, subm by Clark Moore.

CARBONATE SAMPLES OF BIOGENIC ORIGIN

\section{Tiran series}

Samples from Favel Bay, Straights of Tiran (Natl Grid Ref 1100-0735). Coll 1981 by E Spanier, Center Marine Studies, Univ Haifa.

RT-601A.

$1570 \pm 80$

Chicoreus ramosus (gastropod).

RT-601B.

$>\mathbf{3 0 , 0 0 0}$

Fossilized sample of echinoid (sand dollar). 


\section{Achziv series}

Samples from Achziv, 26km N of Haifa (Natl Grid Ref 1596-2718). Coll 1981 by Z Levy and D Neev, Geol Survey Israel, from terrace $7 \mathrm{~m}$ above sea level.

\section{RT-660A.}

Cerastoderma glaucum.

RT-660B.

Unio sp.

RT-660C.

Euthria cornea.
$3240 \pm 180$

$\delta^{13} C=-4.5 \%$

$6000 \pm 170$

$\delta^{13} C=-8.9 \%$

$3640 \pm 160$

$\delta^{13} C=-0.9 \%$

RT-683A. Acre

$P M C=82.0 \pm 2.2$

Aragonitic shell (Euthria cornea). Coll ca 1935 from beach at Acre, $30 \mathrm{~km} \mathrm{~N}$ of Haifa (prebomb sample). Subm 1984 by D Neev.

RT-683B. Tel Aviv

$P M C=89.0 \pm 2.0$

Aragonite shell (Euthria cornea) coll ca 1960, from beach at Tel Aviv (prebomb sample). Subm 1984 by D Neev.

\section{Land snail series}

Land snails were coll by A Karnieli (AK), Desert Research Inst, Sde Boqer, and G A Goodfriend (GG), Weizmann Inst. All samples are from Negev Desert except for RT-674 which is from Jamaica. Data is given in Table 1. Results for live samples are given in percent modern corrected for ${ }^{13} \mathrm{C}$ fractionation, in italics. Natl Grid Refs are given where available; for Jamaican sample, international grid is given. $\delta^{13} \mathrm{C}$ values in parenthesis were estimates by GG. Arad snails (sample RT-746A) were excavated by R Amiran, Hebrew Univ. H Meinis, Zoology Mus Hebrew Univ (HUZM) provided live-collected prebomb land snail shells. Samples RT-732, -741, -744 are from rodent middens. Comment (GG): most fossil snail material was excavated from loessial sediments. Specimens were thoroughly cleaned of all secondary deposits inside and outside. Ages are reported uncorrected for anomalies to which land snails from carbonate substrates are subject (Goodfriend \& Stipp, 1983) which are due to incorporation of carbonate carbon into shell (Goodfriend \& Hood, 1983). Reported ages are thus ca $1000-2000$ yr too old.

\section{HYDROLOGIC SAMPLES}

The Arava samples were coll by R Nativ, Desert Research Inst, Sde Boqer. Galilee and Golan Heights samples were coll by M Stiller, Weizmann Inst, and I Carmi (Carmi, Stiller \& Kaufman, 1985), except for Lake Kin- 


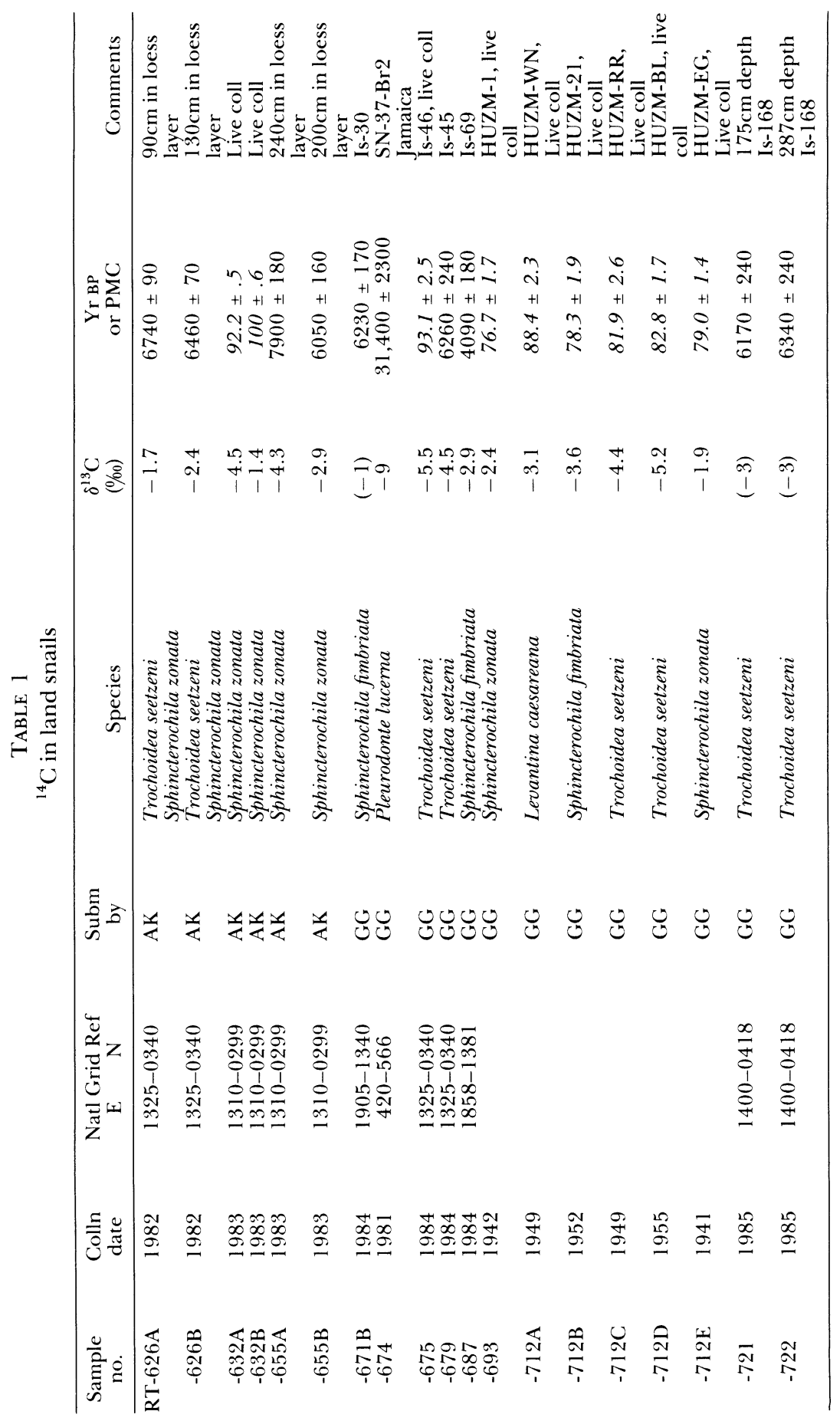


108 Israel Carmi

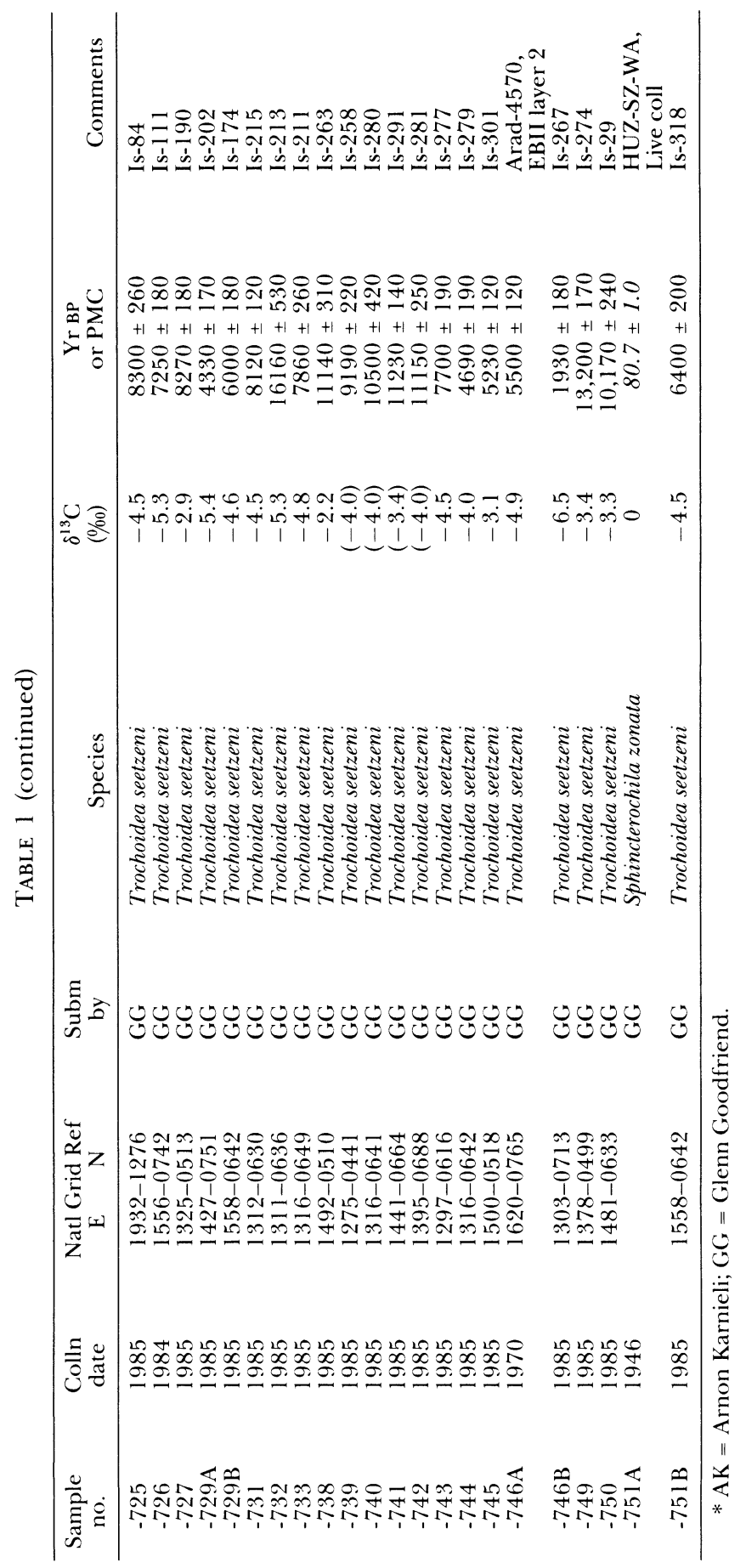


neret samples which were coll by A Kaufman, Weizmann Inst. Mezar samples were coll by G Shaliv TAHAL, Water Planning for Israel Ltd. Dead Sea flood samples were coll by M Stiller. Lowland, Judean Mts and Judean Desert samples were coll by L Kroiteru, Weizmann Inst. Data is given in Table 2.

TABLE 2

${ }^{14} \mathrm{C}$ in hydrologic samples

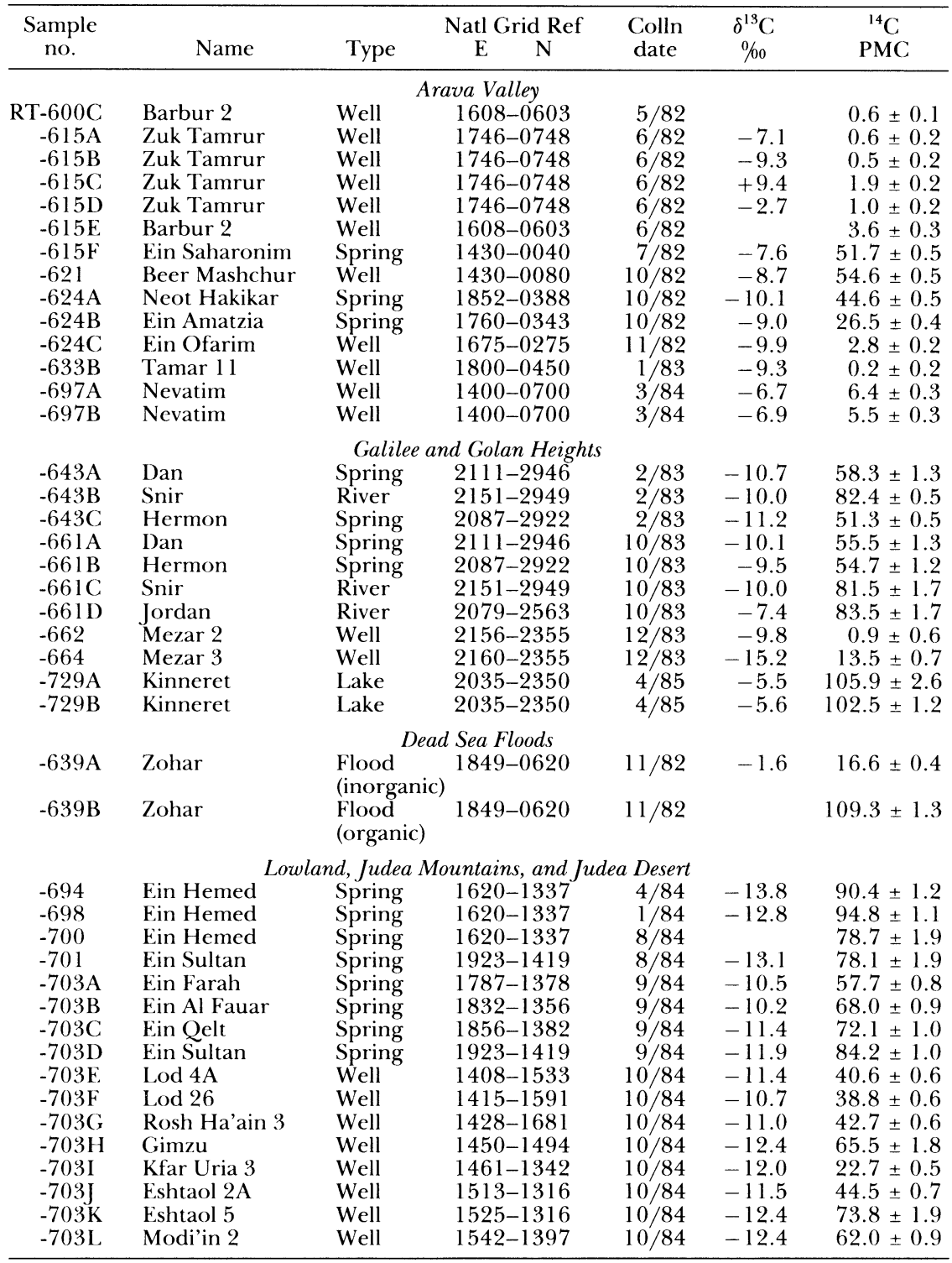


TABLE 2 (continued)

\begin{tabular}{|c|c|c|c|c|c|c|}
\hline $\begin{array}{l}\text { Sample } \\
\text { no. }\end{array}$ & Name & Type & $\begin{array}{c}\text { Natl Grid Ref } \\
\mathrm{E}\end{array}$ & $\begin{array}{l}\text { Colln } \\
\text { date }\end{array}$ & $\begin{array}{c}\delta^{13} \mathrm{C} \\
\% 00\end{array}$ & $\begin{array}{l}{ }^{14} \mathrm{C} \\
\mathrm{PMC}\end{array}$ \\
\hline$-706 \mathrm{~A}$ & Agur 1 & Well & $1422-1254$ & $10 / 84$ & -10.9 & $22.7 \pm 0.5$ \\
\hline$-706 \mathrm{~B}$ & Agur 4 & Well & $1482-1213$ & $10 / 84$ & -11.8 & $24.8 \pm 0.4$ \\
\hline$-706 \mathrm{C}$ & Hartuv 4 & Well & $1501-1287$ & $10 / 84$ & -12.5 & $49.4 \pm 0.7$ \\
\hline$-706 \mathrm{D}$ & Eshtaol 7 & Well & $1513-1307$ & $10 / 84$ & -12.5 & $56.8 \pm 1.4$ \\
\hline$-706 \mathrm{E}$ & Ayalon 3 & Well & $1454-1422$ & $10 / 84$ & -11.9 & $32.6 \pm 0.6$ \\
\hline$-706 \mathrm{G}$ & Ein Karem 6 & Well & $1622-1300$ & $10 / 84$ & -11.6 & $86.8 \pm 2.0$ \\
\hline$-706 \mathrm{H}$ & Ein Karem 1 & Well & $1649-1319$ & $10 / 84$ & -11.5 & $83.1 \pm 1.1$ \\
\hline$-706 \mathrm{I}$ & Jerusalem 6 & Well & $1721-1255$ & $10 / 84$ & -12.0 & $48.4 \pm 0.7$ \\
\hline$-706 \mathrm{~J}$ & Ein Karem 9 & Well & $1664-1347$ & $10 / 84$ & -13.0 & $71.2 \pm 0.9$ \\
\hline$-708 \mathrm{~B}$ & Jerusalem 4 & Well & $1717-1307$ & $11 / 84$ & -10.0 & $41.9 \pm 0.6$ \\
\hline$-708 \mathrm{C}$ & Azariyah & Well & $1766-1320$ & $11 / 84$ & -10.0 & $46.4 \pm 0.8$ \\
\hline$-709 \mathrm{D}$ & Jericho 5 & Well & $1882-1468$ & $11 / 84$ & -12.6 & $37.0 \pm 0.6$ \\
\hline$-709 \mathrm{~F}$ & Jericho 1 & Well & $1909-1408$ & $11 / 84$ & -13.9 & $53.0 \pm 0.8$ \\
\hline$-709 \mathrm{G}$ & Jericho 2 & Well & $1907-1394$ & $11 / 84$ & -11.5 & $44.2 \pm 0.6$ \\
\hline$-713 \mathrm{~A}$ & Ein Farah & Spring & $1787-1378$ & $1 / 85$ & -11.4 & $62.0 \pm 1.7$ \\
\hline$-713 \mathrm{~B}$ & Ein Al Fauar & Spring & $1832-1356$ & $1 / 85$ & -12.9 & $69.0 \pm 0.9$ \\
\hline$-713 \mathrm{C}$ & Ein Qelt & Spring & $1856-1382$ & $1 / 85$ & -13.4 & $79.8 \pm 1.1$ \\
\hline
\end{tabular}

\section{GREENHOUSE SAMPLES}

Samples measured in experiment to estimate incorporation of added $\mathrm{CO}_{2}$ by greenhouse-grown tomato plants. Coll 1983 by Z Enoch, Dept Agric Meteorol, Agric Research Center, Bet Dagan, Israel. Results are given in percent modern carbon (PMC) (Enoch et al, 1984).

\section{RT-637AG.}

$$
\begin{array}{r}
\text { PMC }=115.2 \pm 2.7 \\
\delta^{13} C=-25.2 \% 0
\end{array}
$$

Tomato plant from unenriched greenhouse.

RT-637AE.

$$
\begin{array}{r}
\mathbf{P M C}=\mathbf{6 6 . 7} \pm \mathbf{1 . 5} \\
\delta^{13} C=-37.0^{\circ} \% 0
\end{array}
$$

Tomato plant from greenhouse enriched with tank $\mathrm{CO}_{2}$.

RT-637AG.

$$
\mathbf{P M C}=68.6 \pm 0.5
$$$$
\delta^{13} C=-37.1 \%
$$

Tomato plant from greenhouse enriched with $\mathrm{CO}_{2}$ from burned propane-butane.

\section{DEAD SEA WOOD SAMPLES}

\section{RT-625. Bottom wood}

$$
\mathrm{PMC}=116.9 \pm 2.5
$$

Piece of wood coated with salt crystals, brought up from bottom of Dead Sea at $100 \mathrm{~m}$ bsl (Natl Grid Ref 1890-0960) by mud dredger. Coll 1982 by Y Levy, Geol Survey Israel.

\section{RT-663A. Driftwood}

$$
\delta^{13} C=\begin{aligned}
& \mathbf{3 2 0} \pm \mathbf{8 0} \\
& -12.0 \%
\end{aligned}
$$

Driftwood heavily coated with precipitates and held in place by boul- 
ders, exposed when Dead Sea receded to $-404.5 \mathrm{~m}$ below msl (Natl Grid Ref 1891-1 136). Coll 1983 by Z Klein, Hydrol Service Israel.

\section{RT-683A. Dead Sea Works $(30 \mathrm{~cm})$}

RT-683B. Dead Sea Works $(80 \mathrm{~cm})$

$$
\begin{array}{r}
\mathbf{P M C}=\mathbf{1 0 0 . 0} \pm \mathbf{1 . 2} \\
\delta^{13} C=-22.3 \% 0 \\
\mathbf{P M C}=\mathbf{1 0 3 . 8} \pm \mathbf{1 . 6}
\end{array}
$$

Wood exposed by channel in sediment created by overflow of brine from evaporation ponds of Dead Sea Works (Natl Grid Ref 1905-0507). Coll 1984 by M Magaritz.

\section{RHIZOFOSSIL SAMPLES}

Carbonate filling of root-grooves in Judean Desert. Coll 1983-4 by A Danin, Dept Botany, Hebrew Univ, Jerusalem (Danin, Wieder \& Magaritz, in press). $\delta^{13} \mathrm{C}$ values in parentheses were estimated by M Magaritz.

\section{RT-646A. Maaleh Adumim}

(Natl Grid Ref 1700-1325) from depth 2m.

\section{RT-646B. Anatot}

(Natl Grid Ref 1767-1369).

\section{RT-678A. Maaleh Adumim}

(Natl Grid Ref 1700-1325) from depth 2m.

RT-678C.

(Natl Grid Ref 1767-1369) from depth 2 to $3 \mathrm{~m}$.

$$
\begin{array}{r}
\mathbf{3 0 , 5 0 0} \pm \mathbf{9 0 0} \\
\delta^{13} C=-11.5 \%
\end{array}
$$

$\mathbf{2 9 , 8 0 0} \pm \mathbf{8 0 0}$ $\delta^{13} C=-10.8 \%$

$$
31,400 \pm 1200
$$$$
\delta^{13} \mathrm{C}=-11.0 \%
$$

$$
\begin{array}{r}
>\mathbf{4 4 , 0 0 0} \\
\left(\delta^{13} C=-11 \% 0\right)
\end{array}
$$

\section{LISAN SAMPLES}

\section{Lisan series}

Samples coll near boundaries of late Pleistocene Lisan Lake, precursor of present Dead Sea. Coll 1982 by B Buchbinder, Geol Survey Israel (Buchbinder, 1981).

\section{RT-613A. Hirbet Samra}

Lisan stromatolite from near Jericho (Natl Grid Ref 1950-1460).

RT-613B. Nahal Mor

$\mathbf{1 7 , 6 0 0} \pm \mathbf{5 0 0}$

Lisan stromatolite (Natl Grid Ref 1850-990). 
RT-613C. Zohar

Laminar tufa (Natl Grid Ref 1843-0630).

RT-613D. Zohar

Postular tufa (Natl Grid Ref 1845-0630).

$$
\begin{array}{r}
>40,000 \\
\delta^{13} C=+0.8 \%
\end{array}
$$$$
>\mathbf{4 0 , 0 0 0}
$$$$
\delta^{13} C=+0.9 \%
$$

\section{Nahal Amatzyah series}

Oolite samples consisting of calcite and aragonite. Ages are given in Druckman, Magaritz \& Sneh (in press).

RT-620A.

$\mathbf{P M C}=\mathbf{8 . 5 0} \pm .25$

$\delta^{13} C=-4.1 \%$

Oolite (Natl Grid Ref 1765-0353). Coll 1982 by M Magaritz.

RT-620B.

$\mathbf{P M C}=\mathbf{5 . 6} \pm .27$

$\delta^{13} C=+1.5 \%$

Oolite (Natl Grid Ref 1776-0378). Coll 1982 by M Magaritz.

RT-635.

$14,600 \pm 200$

$\delta^{13} C=-25.6 \%$

Organic matter in clay matrix (Natl Grid Ref 1768-0367). Coll 1982 by M Magaritz.

HULA CORE SAMPLES

Dates from core coll in drilling operation at Hula Basin, $\mathrm{N}$ Israel (Natl Grid Ref 1264-0614). Subm 1980 by M Magaritz. In age calculation, $\delta^{13} \mathrm{C}=$ $-25 \%$ was assumed (Kafri, Kaufman \& Magaritz, 1983).

RT-610A.

$20,940 \pm 390$

Depth $46.5 \mathrm{~m}$.

RT-610B.

$34,000 \pm 1700$

Depth $55.0 \mathrm{~m}$.

\section{CALCITE NODULES SAMPLES}

Calcite nodules from loess sections, Negev, measured to date environmental changes in upper Pleistocene along desert boundary (Magaritz, in press).

\section{Netivot series}

Section at Netivot, Negev near Beer Sheba (Natl Grid Ref 1110-0930). Coll 1982 by M Magaritz.

\section{RT-604C.}

$7240 \pm 90$

Depth $80 \mathrm{~cm}$. 
RT-604D.

Depth $1 \mathrm{~m}$.

RT-619B.

Depth $7 \mathrm{~m}$.

RT-629A.

Depth $5.5 \mathrm{~m}$.

RT-629B.

Depth $7.5 \mathrm{~m}$.
$13,630 \pm 100$

$\delta^{13} C=-3.8 \% 0$

$27,900 \pm 660$

$\delta^{13} C=-11.9 \%$

$35,000 \pm 1500$

$\delta^{13} C=-9.3 \%$ o

$\mathbf{2 4 , 4 0 0} \pm \mathbf{4 5 0}$

$\delta^{13} C=-11.1 \%$

\section{Ramat Hovav series}

Coll 1982 by M Magaritz, from 7 loci along Nahal Sekher, 34km SE of Netivot sec.

RT-604A.

$\mathbf{1 0 , 5 0 0} \pm \mathbf{1 3 0}$

RT-604A.

$\delta^{13} C=-3.2 \%$

Lacustrine sediment (Natl Grid Ref 1316-0577) from depth 7m.

RT-604B.

$11,680 \pm 140$

$\delta^{13} C=-4.1 \%$

Lacustrine sediment (Natl Grid Ref 1316-0577) from depth $6 \mathrm{~m}$.

RT-606A.

$25,900 \pm 400$

$\delta^{13} C=-1.7 \%$

Calcite nodules (Natl Grid Ref 1308-0575) from depth 4.8m.

RT-606B.

$\mathbf{3 0 , 0 0 0} \pm \mathbf{8 0 0}$

$\delta^{13} C=-3.9 \%$

Calcite nodules (Natl Grid Ref 1301-0577) from depth ca $1 \mathrm{~m}$.

RT-606D.

Calcite nodules (Natl Grid Ref 1284-0591).

RT-607A.

Calcite nodules (Natl Grid Ref 1286-0591).

RT-607B.

Calcite nodules (Natl Grid Ref 1286-0591). $\mathbf{2 9 , 0 0 0} \pm \mathbf{7 0 0}$ $\delta^{13} C=-2.8 \%$

$\mathbf{2 5 , 9 0 0} \pm \mathbf{5 0 0}$ $\delta^{13} C=-3.2 \%$

$21,900 \pm 300$

$\delta^{13} C=-3.3 \%$ 
$16,100 \pm 270$

RT-607D.

$\delta^{13} C=-1 \%$ o

Calcite nodules (Natl Grid Ref 1308-0575).

RT-608A.

Calcite nodules (Natl Grid Ref 1264-0614).

\section{RT-608B.}

Calcite nodules (Natl Grid Ref 1264-0614).

\section{RT-608C.}

Calcite nodules (Natl Grid Ref 1264-0614). $>\mathbf{3 5 , 0 0 0}$

$\delta^{13} C=-1.0 \%$

$>\mathbf{3 5 , 0 0 0}$

$\delta^{13} C=-0.7 \%$

$9300 \pm 100$

$\delta^{13} C=-2.4 \%$

\section{REFERENCES}

Avner, U, 1984, Ancient cult sites in the Negev and Sinai deserts: Tel Aviv, v 11, p 115-131.

Buchbinder, B, 1981, Morphology, microfabric and origin of stromatolites of the Pleistocene precursor of the Dead Sea, Israel, in Monty, C, ed, Phanerozoic stromatolites: Berlin, Springer Verlag, p 181-195.

Carmi, I, Noter, Y and Schlesinger, R, 1971, Rehovot radiocarbon measurements I: Radiocarbon, v 13, no. 2, p 412-419.

Carmi, I, Stiller, $\mathrm{M}$ and Kaufman, A, 1985, The effect of atmospheric ${ }^{14} \mathrm{C}$ variations on the ${ }^{14} \mathrm{C}$ levels in the Jordan River system: Radiocarbon, v 27, no. 2B, p 305-313.

Danin, A, Wieder, $M$ and Magaritz, $M$, in press, Rhizofossils and root-grooves in the Judean Desert and their paleo-environmental significance: Paleogeog Paleoclimat Paleoecol.

Druckman Y, Magaritz, M and Sneh, A, in press, Leaching and cementation of Late Pleistocene oolites from Lake Lisan, Dead Sea rift, Israel: Jour Sed Petrol.

Enoch, Z H, Carmi, I, Rounick, IS and Magaritz, M, 1984, Use of carbon isotopes to estimate incorporation of added $\mathrm{CO}_{2}$ by greenhouse-grown tomato plants: Plant Physiol, v 76, p $1083-1085$

Goodfriend, G A and Stipp, J J, 1983, Limestone and the problem of radiocarbon dating of land-snail shell carbonate: Geology, v 11, p 575-577.

Goodfriend, G A and Hood, D G, 1983, Carbon isotope analysis of land snail shells: implications for carbon sources and radiocarbon dating: Radiocarbon, v 25, no. 3, p 810-830.

Henry, D, 1982, The prehistory of southern Jordan and relationships with the Levant: Jour Field Archeol, v 9, no. 4, p 417-444.

Hohfelder, R, 1981, The ancient harbor of Cesarea Maritima: Archaeology, v 84, p 56-60.

Kafri, U, Kaufman, A and Magaritz, M, 1983, The rate of Pleistocene subsidence and sedimentation in the Hula basin as compared with those of other time spans in other Israeli tectonic regions: Earth Planetary Sci Letters, v 65, p 126-132.

Levy, T E, 1983, The emergence of specialized pastoralism in the southern Levant: World Archaeol, v 15, p 15-36.

Magaritz, M, in press, Environmental changes in the upper Pleistocene along the desert boundary, southern Israel: Paleogeog Paleoclimat Paleoecol.

Rosen S, 1984, Kvish Harif: preliminary investigations at a late Neolithic site in the Central Negev: Paleorient, v 10, p 111-121.

Wachsmann, S and Raveh, K, 1984, A concise history of Dor/Tantura: Internatl Jour Nautical Archaeol Underwater Exploration, v 13, p 223-241.

Yogev, O, 1983, A fifth millennium BCE sanctuary in the 'Uvda Valley: Qadmoniot, v 16, no. 4, p 118-122 (in Hebrew). 